The International Journal of Multimedia \& Its Applications (IJMA) Vol.6, No.5, October 2014

\title{
LEADER FOLLOWER FORMATION CONTROL OF GROUND VEHICLES USING DYNAMIC PIXEL COUNT AND INVERSE PERSPECTIVE MAPPING
}

\author{
S.M.Vaitheeswarana, Bharath.M.K, Ashish Prasad and Gokul.M \\ Aerospace Electronics and Systems Division, CSIR-National Aerospace laboratories, \\ HAL Airport Road, Kodihalli, Bengaluru, 560017 India
}

\begin{abstract}
This paper deals with leader-follower formations of non-holonomic mobile robots, introducing a formation control strategy based on pixel counts using a commercial grade electro optics camera. Localization of the leader for motions along line of sight as well as the obliquely inclined directions are considered based on pixel variation of the images by referencing to two arbitrarily designated positions in the image frames. Based on an established relationship between the displacement of the camera movement along the viewing direction and the difference in pixel counts between reference points in the images, the range and the angle estimate between the follower camera and the leader is calculated. The Inverse Perspective Transform is used to account for non linear relationship between the height of vehicle in a forward facing image and its distance from the camera. The formulation is validated with experiments.
\end{abstract}

\section{KEYWORDS}

Vision based range and angle measurement, dynamic pixel count controller, inverse mapping projection.

\section{INTRODUCTION}

Unmanned Systems for autonomous navigation requires some form of situational awareness of the robot's environment. This awareness is achieved commonly through different sensors such as high end electro-optic cameras, traditional stereo cameras, scanning laser range finders, or some combination of these sensors. However, these sensors provide an overwhelming amount of data and come at the expense of additional computational and increased costs to the system. In this paper, a low cost, low computation, vision solution to situational awareness in the framework of a formation control convoy formation problem for autonomous ground vehicles is proposed. The Vision system uses the traditional monocular cameras but differs in its computational method[1,2,3]. The traditional camera generates tens of thousands of data points creating a very fine map of the environment, but does not provide any insight into the recognition of objects within the scene. The simplified vision system presented here in the paper works by first recognizing the leader as a high contrast symbol within the camera images. The correlation problem is reduced from tens of thousands of pixels to a set of feature points and in some cases just one feature point. A vector for the relative position of the robot to the scene feature is 
The International Journal of Multimedia \& Its Applications (IJMA) Vol.6, No.5, October 2014

calculated based on the dynamic pixel count in the images and is used to generate the motion commands for the follower robot. The process is repeated iteratively until the robot is correctly localized. A second advantage of the approach is that the algorithm used is heavily researched, well documented and several fast open source algorithms already exist. A third contribution to the paper is in the distance measurement scheme based on dynamic pixel measurements. If the leader under measurement is not perpendicular to the optical axis, a relationship between the displacement of the camera movement and the difference in pixel counts is used to measure the photographing distance and inclination angle to get the leader position. lastly the angle of view under which a scene is acquired and the distance of the objects from the camera, namely the perspective effect, contributes different information content to each pixel of an image. To cope with this problem a geometrical transform Inverse Perspective Mapping, (IPM) [4], is used in this paper. This allows removing the perspective effect from the acquired image, remapping it into a new 2-dimensional domain (the remapped domain).

\section{PROBLEM STATEMENT}

Leader-follower formation of non-holonomic ground vehicles is assumed. The follower ground vehicle is equipped with a camera to obtain its bearing and position information with respect to the leader robot based on pixel measurement count in the images. Vehicle kinematics obeys the bicycle model. The controller's goal is to keep a desired position and orientation with respect to the leader. The problem is to find the relative position and orientation with respect to the leader so that it can be provided as feedback to the control loop. Accurate distance measurements are crucial, as the follower robot will be planning its path based upon the data from the vision system. It is important for the hardware and software to have a fast update rate with a minimum of delay, as both can directly affect the dynamic performance of the control system for the camera.

With these requirements in mind, this work presents a system selected for their individual characteristics and complementary nature: 1) A color detector working in the RGB image space, and 2) Inverse Perspective Mapping (IPM) to create a top down view of the image 3) Angle and Distance Estimation of the leader and follower based on pixel counts in the images.

\section{Methodology}

This section describes the algorithm used to identify a leader object in the sequence of images from a color video camera mounted on the follower vehicle to estimate the range and bearing to the target.

\subsection{Color detection}

The color detector identifies areas that match the leader's color characteristics using a number of steps shown below:

1. The image is separated into its constituent Red Green and Blue channels. The luminance $f_{1}(x, y)$ of the pixel $(x, y)$ is described as [5]:

$$
f_{1}(x, y)=0.299 R_{(x, y)}+0.578 G_{(x, y)}+0.114 B_{(x, y)}
$$


The International Journal of Multimedia \& Its Applications (IJMA) Vol.6, No.5, October 2014

2. Taking one of the RGB channels, the algorithm calculates the average pixel intensity from the area immediately in front of the vehicle.

3. A binarization [5] is performed using the average value from step 2 as the threshold. The pixels similar to surface (path) are thresholded to white and others are threshholded to black, where 1 signifies a road pixel, and a 0 is a non-road pixel.

4. The steps 2 and 3 are repeated for the other channels (Green and Blue).Morphological image processing operations [5], such as dilation, erosion, opening and closing are used after the process of image segmentation, in order to enable the underlying shapes to be identified and optimally reconstruct the image from their noisy precursors. Connected-components that share similar pixel intensity values are identified and then connected with each other. The connected-component labelling scans an image and groups pixels into one or more components according to pixel connectivity. Once all groups are determined, each pixel is labelled with a grey level on the basis of the component.

5. Finally, the algorithm merges the 3 new binary images into a new RGB image

\subsection{Inverse perspective mapping (IPM)}

IPM is a mathematical technique whereby a coordinate system may be transformed from one perspective to another. In this case we use IPM to remove the effects of perspective distortion of the road surface in the forward facing image, to an undistorted top-down view as shown in Fig. (1). As can be seen lines that appear to converge near the horizon are made parallel To get a bird eye's view image, a perspective transform relating the pixel $(\mathrm{u}, \mathrm{v})$ values seen by the camera in the original image is obtained through a transformation matrix as shown in figure.

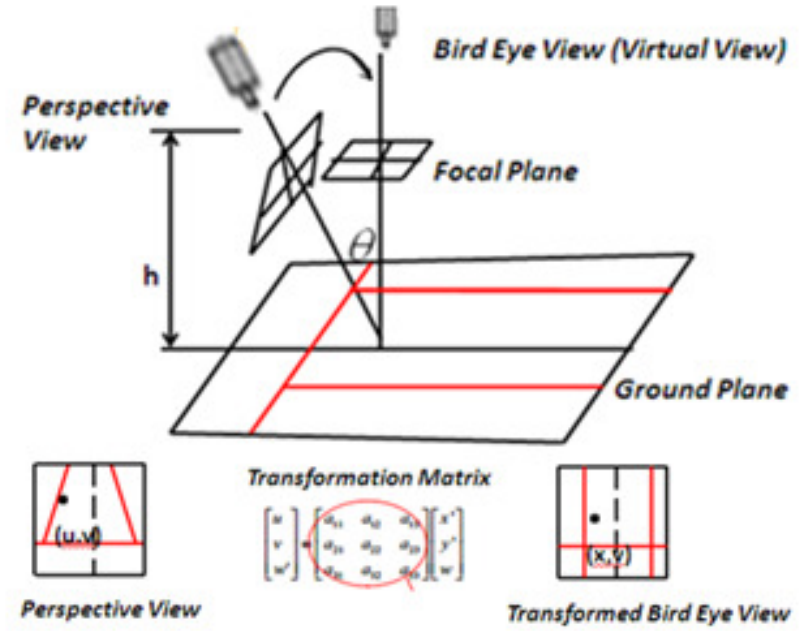

Figure 1. IPM Transformation

Transforming the image in this manner removes the non linearity of distances and the orientation. We can focus our attention on only a sub-region of the input image, which helps in reducing the run time considerably. In order to create the top-down view, we first need to find the mapping of a point on the road to its projection on the image surface. To get the IPM of the input image, we assume a flat road, and use the camera intrinsic (focal length and optical center) and extrinsic ( $\theta$ 
The International Journal of Multimedia \& Its Applications (IJMA) Vol.6, No.5, October 2014

and height above ground) parameters to perform this transformation. We start by defining a world frame $\left\{\mathrm{F}_{\mathrm{W}}\right\}=\left\{\mathrm{X}_{\mathrm{W}}, \mathrm{Y}_{\mathrm{W}}, \mathrm{Z}_{\mathrm{W}}\right\}$ centered at the camera optical center, a camera frame $\left\{\mathrm{F}_{\mathrm{C}}\right\}=$ $\left\{\mathrm{X}_{\mathrm{C}}, \mathrm{Y}_{\mathrm{C}}, \mathrm{Z}_{\mathrm{C}}\right\}$, and an image frame $\left\{\mathrm{F}_{\mathrm{i}}\right\}=\{\mathrm{u}, \mathrm{v}\}$ as shown in Fig.(1). We assume that the camera frame $\mathrm{X}_{\mathrm{C}}$ axis stays in the world frame $\mathrm{X}_{\mathrm{W}} \mathrm{Y}_{\mathrm{W}}$ plane. The height of the camera frame above the ground plane is $\mathrm{h}$. Starting from a point in the image plane iP $=\{\mathrm{u}, \mathrm{v}, 1,1\}$, it can be shown that its projection on the road plane can be found by applying the homogeneous transformation.

This mapping is expressed as [6]:

$$
{ }_{i} P=\{u, v, 1,1\}=[K][T][R](x, y, z, 1)
$$

Where $\mathrm{R}$ is the rotation matrix:

$$
R=\left[\begin{array}{cccc}
1 & 0 & 0 & 0 \\
0 & \cos \theta & -\sin \theta & 0 \\
0 & \sin \theta & \cos \theta & 0 \\
0 & 0 & 0 & 1
\end{array}\right]
$$

$\mathrm{T}$ is the translation matrix:

$$
T=\left[\begin{array}{llll}
1 & 0 & 0 & 0 \\
0 & 1 & 0 & 0 \\
0 & 0 & 1 & -h / \sin \theta \\
0 & 0 & 0 & 1
\end{array}\right]
$$

And $\mathrm{K}$ is the camera parameter matrix:

$$
K=\left[\begin{array}{lllll}
f * k u & s & \text { uo } & w \\
0 & f * k v & \text { vo } & 0 \\
0 & 0 & 1 & 0
\end{array}\right]
$$

Here, $\mathrm{f}$ is the focal length of the camera and $(k u * k v)$ is the aspect ratio of the pixels, $u o$ and vo the camera centre coordinates. Fig. (2) shows a typical IPM sample. The leader vehicle is extracted using object detection process and a background subtraction procedure from the transformed bird eye view images of the follower.

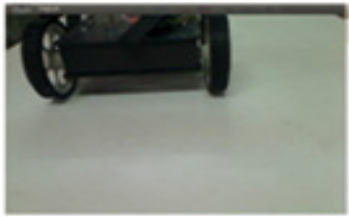

(a)

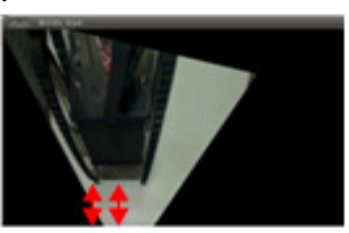

(b)

Figure 2. IPM Sample (a) Perspective (frontal) View; (b) Bird Eye View 
The International Journal of Multimedia \& Its Applications (IJMA) Vol.6, No.5, October 2014

A pose estimation of the leader vehicle is performed in order for the follower vehicle to navigate. Only the area directly visible in front of the host vehicle is analyzed. Cropping is performed on the road segmented image, in order to get the region of interest which provides us the distance and angle of orientation of leader in term of pixels. The two red lines in Fig 2(b) indicate the distance of the leader vehicle which is being taken as two cropped images for computation as shown in Fig.(2).

\subsection{Target Localization based on pixel count [6-9]}

The idea of localization is from the triangular relationship, shown in Fig. 3. In this, we first capture an image of the leader incorporating a known-dimension rectangle, and then the proportion relationship between the real-dimension and the image-dimension of the rectangle is found. According to the proportion relationship, the distance $d$ is easily calculated $[6,7,8,9]$.

In this section we outline the methodology used for range and angle estimation based on the pixel count number variation in images for the purpose of localization of the leader in the images. For this we first capture an image incorporating a known-dimension trapezoid, and then the proportion relationship between the real-dimension and the image-dimension of the trapezoid are found. In this we have two cases: a. the targeted leader vehicle lying on the plane perpendicular to the optical axis of the follower vehicle camera, b. the targeted leader vehicle lies on an oblique tangent plane with respect to the optical axis of the follower vehicle camera.

\section{Case a: Leader perpendicular to the optical axis of the follower camera:}

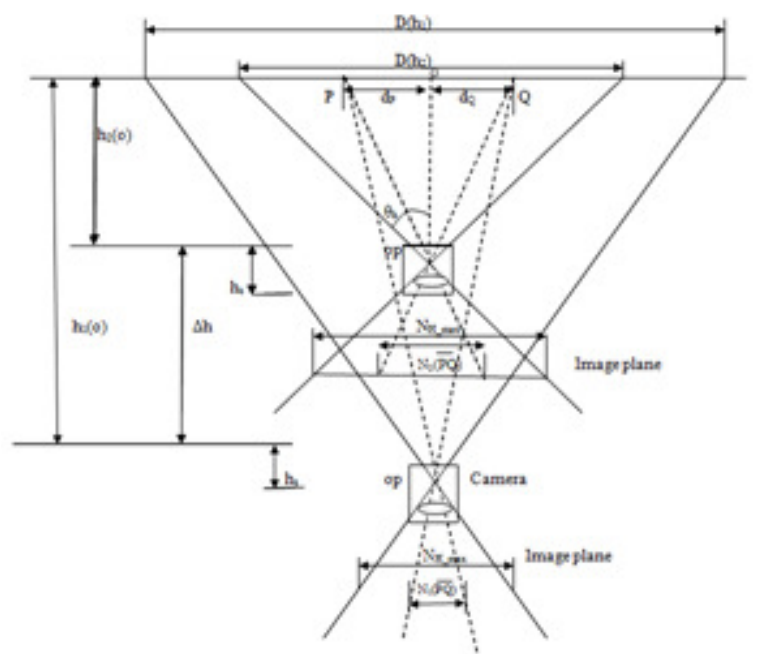

Figure 3. Pixel Count Measurement Geometry using Bird Eye View Image for perpendicular Case

As shown in Fig.(3) the target plane is assumed to be perpendicular to the optical axis and the relationship between distance and variation of pixel counts at different viewing distances are obtained using Figure and geometry. The target plane formed by the leader vehicle is represented by points $\mathrm{P}, \mathrm{O}, \mathrm{Q}$. A simple relationship between the change in leader follower distance $\Delta$, and the difference in pixel counts between the reference points in the images at different viewing distances, are obtained with the assumption that the distance between reference points $\mathrm{P}$ and $\mathrm{Q}$ will not change. We have the following relationship between pixel counts and distances: 
The International Journal of Multimedia \& Its Applications (IJMA) Vol.6, No.5, October 2014

$$
\begin{aligned}
& \frac{d(\overline{P Q})}{n_{1}(\overline{P Q})}=\frac{d\left(h_{1}\right)}{n_{\max }} \\
& \frac{d(\overline{P Q})}{n_{2}(\overline{P Q})}=\frac{d\left(h_{2}\right)}{n_{\max }}
\end{aligned}
$$

Where, points $\mathrm{P}$ and $\mathrm{Q}$ is the target plane distance. $n_{1}(\overline{P Q})$ and $n_{1}(\overline{P Q})$ are the pixel counts between points $\mathrm{P}$ and $\mathrm{Q}$ on the image plane at distances $h_{1}(0)$ and $h_{2}(0)$ respectively. $\Delta h$ is the change in distance between the leader and follower positions. $h_{5}$ is the distance between the optical origin (OP) and the front end of the camera on the follower. From (1) and (2), we have:

$$
\frac{d\left(h_{1}\right)}{d\left(h_{2}\right)}=\frac{n_{2}(\overline{P Q})}{n_{1}(\overline{P Q})}
$$

Because of the displacement, $\Delta h=h_{1}(0)-h_{2}(0)$, we have:

$$
\frac{n_{2}(\overline{P Q})}{n_{1}(\overline{P Q})}=\frac{d\left(h_{1}\right)}{d\left(h_{2}\right)}=\frac{h_{1}(0)+h_{s}}{h_{2}(0)+h_{S}}
$$

Substituting $h_{1}(0)=h_{2}(0)+\Delta h$ into (4), viewing distances $h_{1}(0)$ and $h_{2}(0)$ of the leader on the perpendicular plane can be obtained as:

$$
\begin{aligned}
& h_{1}(O)=\frac{n_{2}(\overline{P Q})}{n_{2}(\overline{P Q})-n_{1}(\overline{P Q})} * \Delta h-h_{S} \\
& o r \\
& h_{2}(O)=\frac{n_{1}(\overline{P Q})}{n_{2}(\overline{P Q})-n_{1}(\overline{P Q})} * \Delta h-h_{s}
\end{aligned}
$$

Also, distance between points A and B can be obtained as:

$$
\begin{aligned}
\overline{A B} & =\frac{2\left(h_{2}(0)+h_{S}\right) * \tan \theta_{H}}{N_{H_{-} \max }} * N_{2}(\overline{A B}) \\
& =\frac{2\left(h_{1}(0)+h_{S}\right) * \tan \theta_{H}}{N_{H_{-} \max }} * N_{1}(\overline{A B})
\end{aligned}
$$

where, $\theta_{\mathrm{H}}$ is the maximal horizontal view angle of the camera and $\mathrm{N}_{\mathrm{H}-\max }$ is the maximal pixels in a horizontal scan line of an image frame, which is fixed and known as a priori irrelevant of photographing distances. As a result of this relationship, the distance between an arbitrarilyselected point $\mathrm{P}$ and the origin $\mathrm{O}$ can also be represented as follows: 
The International Journal of Multimedia \& Its Applications (IJMA) Vol.6, No.5, October 2014

$$
\begin{aligned}
\overline{P Q} & =\frac{2\left(h_{2}(O)+h_{S}\right) * \tan \theta_{H}}{n_{H_{-} \max }} * n_{2}(\overline{P Q}) \\
& =\frac{2\left(h_{1}(O)+h_{S}\right) * \tan \theta_{H}}{n_{H_{-} \max }} * n_{1}(\overline{P Q})
\end{aligned}
$$

The above-mentioned relationship is only valid for leader objects or planes that are perfectly perpendicular to the optical axis.

\section{Case b: Leader oblique to the optical axis of the follower camera:}

Fig. (4) shows the configuration of the proposed image-based system for measuring the leader position on an oblique surface based on variation of pixel counts, where an incline angle $\theta_{m}$ exists between the oblique and perpendicular planes. Virtual planes are introduced to address the problem to measure the incline angle $\theta_{m}$. Thus, distances between the camera and the virtual planes for different positions can be obtained as:

$$
\begin{aligned}
& h_{1}(P)=\frac{n_{2}(P)}{n_{2}(P)-n_{1}(P)} * \Delta h-h_{S} \\
& h_{2}(P)=\frac{n_{1}(P)}{n_{2}(P)-n_{1}(P)} * \Delta h-h_{S}
\end{aligned}
$$

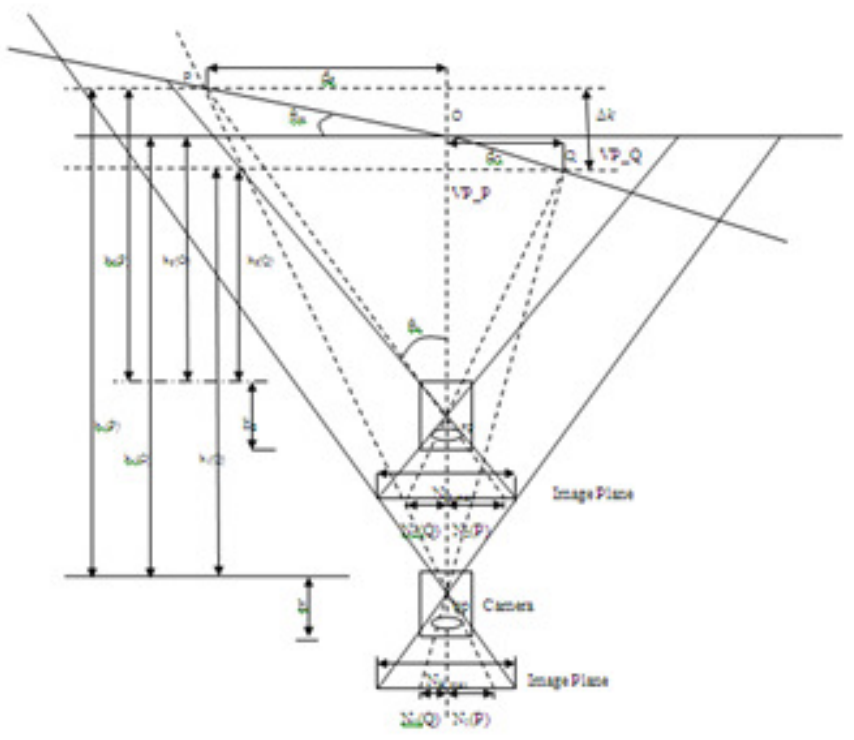

Figure 4. Pixel Count Measurement Geometry using Bird Eye View Image for Oblique Case 
The International Journal of Multimedia \& Its Applications (IJMA) Vol.6, No.5, October 2014

$$
\begin{aligned}
& h_{1}(Q)=\frac{n_{2}(Q)}{n_{2}(Q)-n_{1}(Q)} * \Delta h-h_{S} \\
& h_{2}(Q)=\frac{n_{1}(Q)}{n_{2}(Q)-n_{1}(Q)} * \Delta h-h_{S}
\end{aligned}
$$

where, $h_{1}(P)$ and $h_{1}(Q)$ are distances from the camera at position 1 to virtual plane VP_P and virtual plane VP_Q, respectively. $h_{2}(P)$ and $h_{2}(Q)$ are distances from the camera at position 2 to virtual plane VP_P and virtual plane VP_Q, respectively. $n_{n}(P)$ is the pixel counts in the image plane for point $\mathrm{P}$ when image is taken at position ${ }^{n}$. From (9), (10), (11), and (12), we have:

$$
\begin{aligned}
& h_{1}(P)-h_{1}(Q)=h_{1}(P)-h_{1}(Q)=\Delta k \\
& \Delta k=\left(d_{P}+d_{Q}\right) \tan \theta_{m} \\
& \theta_{m}=\tan ^{-1} \frac{\Delta k}{d_{P}+d_{Q}}
\end{aligned}
$$

where, $\Delta K$ is the distance between the virtual planes VP_P and VP_Q , and $\theta_{\mathrm{m}}$ is the incline angle. $d_{P}$ and $d_{Q}$ represent the distance from point $P$ and point $Q$ to the optical axis, respectively, which can be expressed as follows:

To this end, the distance from the optical origin at positions 1 and 2 to the oblique plane intersecting the optical axis can be expressed as:

$$
\begin{aligned}
& h_{1}(O)=h_{1}(P)-d_{P} \tan \theta_{m}=h_{1}(Q)+d_{Q} \tan \theta_{m} \\
& h_{2}(O)=h_{2}(Q)-d_{P} \tan \theta_{m}=h_{2}(Q)+d_{Q} \tan \theta_{m}
\end{aligned}
$$

and the distance between two arbitrary point $\mathrm{P}$ and $\mathrm{Q}$ as $\mathrm{D}(\overline{\mathrm{PQ}})$ is derived as $D(\overline{P Q})=\left|d_{P}+d_{Q}\right| \sec \theta_{m}$

\subsection{Vehicle kinematics and control}

The goal of the vehicle controller is to use the leader's range and bearing from the vision system to force the follower vehicle to follow the leader's path. The controller designed to follow the path the leader is delayed by an arbitrary following time, ${ }^{\tau}$ (typically 10 seconds or so).

If $(\mathrm{x}, \mathrm{y})$ is the global position of the follower and $\left(\mathrm{x}_{0}, \mathrm{y}_{0}\right)$ is the position of the leader, the controller attempts to make $(\mathrm{x}(\mathrm{t}), \mathrm{y}(\mathrm{t}))$ track $\left(\mathrm{x}_{0}(\mathrm{t}-\tau), \mathrm{y}_{0}(\mathrm{t}-\tau)\right)$ For simplicity, the delayed leader position is defined $\left(\mathrm{x}_{\mathrm{d}}(\mathrm{t}), \mathrm{y}_{\mathrm{d}}(\mathrm{t})\right)$. The position of the leader vehicle, $\left(\mathrm{x}_{0}, \mathrm{y}_{0}\right)$, is determined using the camera vision data relative to the current follower position, as described in Section. 
The International Journal of Multimedia \& Its Applications (IJMA) Vol.6, No.5, October 2014

The controller uses a bicycle model for a kinematic model of the vehicle, which is commonly used in vehicle following applications. Referring to Fig. (5), ( $x, y)$ is the follower position, $\mathrm{v}_{\mathrm{c}}$ is the commanded speed, and $\gamma_{c}$ is the commanded steering. The control laws are given as

$$
\begin{gathered}
v_{c}=v_{d}+k_{p, e 1} e 1, \quad k_{p, e 1}>0 \\
\gamma_{c}=k_{p, e_{2}}{ }^{e+k_{p, e_{\theta 1}} e_{\theta}, \quad k_{p, e_{2}} e_{2,}{ }^{k}{ }_{p, e_{\theta 1}} e_{\theta}>0}
\end{gathered}
$$

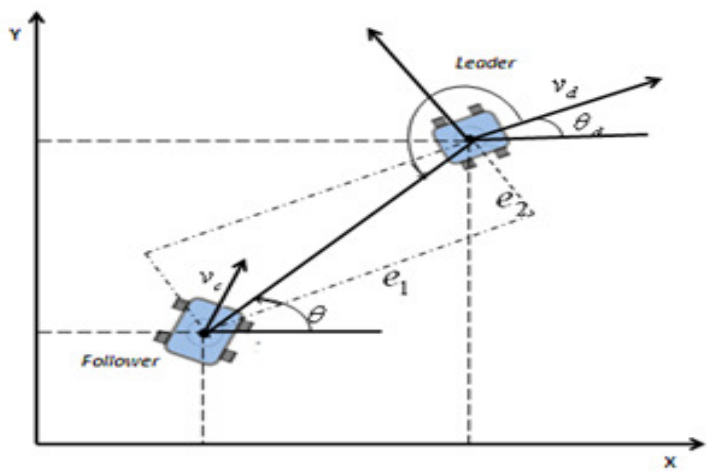

Figure 5. Leader Follower Geometry and Co-ordinate system

$$
\begin{aligned}
& e_{1}=\left(x_{d}-x\right) \cos \theta+\left(y_{d}-y\right) \sin \theta \\
& e_{2}=-\left(x_{d}-x\right) \sin \theta+\left(y_{d}-y\right) \cos \theta \\
& e_{\theta}=\theta_{d}-\theta
\end{aligned}
$$

\section{EXPERIMENTAL SET UP AND IMPLEMENTATION}

In order to show the effectiveness of the algorithms, experiments were performed on a set up consisting of two ground vehicles in a convoy formation using autonomous control for the master and vision control for the slave. The robot vehicle frame and chassis is a model manufactured by Robokit, INDIA. It has a differential 4-wheeled robot base with two wheels at the front and the two wheels are at the back. The electronics is developed in-house. The vehicle uses four DC motors (geared) for driving the four wheels independently. The DC motors are controlled by Atmega microcontroller and controller board is shown in figure. For this purpose, the $\mathrm{C}$ language, together with the OpenCV library of is used.

The follower tracks the remotely controllable leader moving in an arbitrary trajectory, while keeping a predefined inter-distance. The control system has two parts - platform inter-distance control and control of follower orientation with respect to the leader. The follower orientation control provides actions needed for follower to face the leader, which is accomplished when the leader is located in the center of image captured by the follower. The distance between the leader and the follower is obtained from the leader size in current frame. An increase in the platform inter-distance causes the decrease in the platform size in an image, and vice versa. This dependency has been experimentally obtained. 
The International Journal of Multimedia \& Its Applications (IJMA) Vol.6, No.5, October 2014

The difference between current and desired platform inter-distance causes the follower forward/backward motion, while the leader's displacement from the image center causes the follower rotation until the leader appears in the image center.

Three different postures (angles of view) of the platform at three separation distances have been examined. For each posture and distance (9 cases) 90 frames have been processed (15 fps). Determination of the position and orientation of the leader is achieved by estimating its distance and relative orientation with regard to the follower using the procedure outlined in Section III.

Only the area directly visible in front of the host vehicle is analyzed. Cropping is performed on the road segmented image, in order to get the region of interest which provides us the distance and angle of orientation of leader in term of pixels.

In case of front view (straight) as shown in fig 6(b) and for the different distances considered results show very good localization and following capabilities. For the left and right views shown in Fig (6a and 6c) the results are also in good agreement indicating the validity of the design and implementation. Fig.(7) shows typical snapshots capturing the faithful change in movements in straight line and orientation of the follower vehicle as demanded indicating the validity of the approach and method used in the paper.

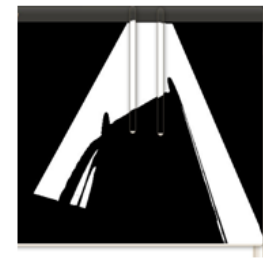

(a)

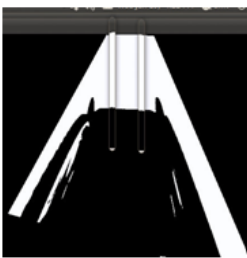

(b)

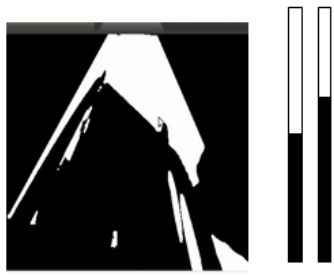

(c) (d)

Figure 6. Distance and orientation of the leader vehicle. (a) left orientation 350, (b) straight movement 00,(c) Right orientation 500, (d) cropped version used for pixel count

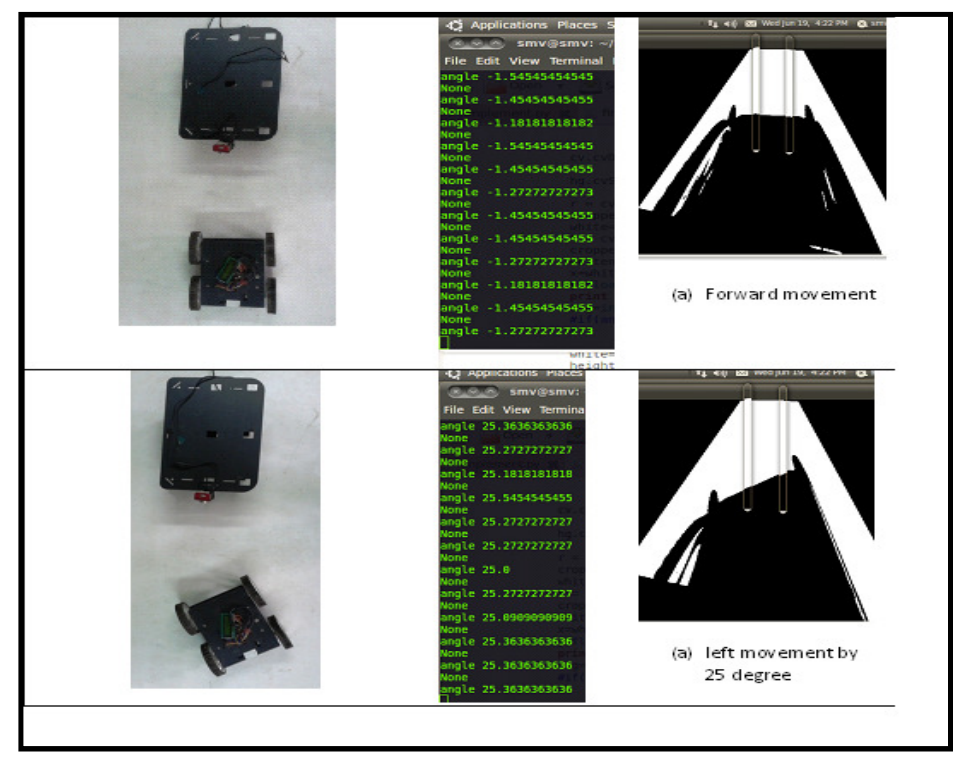

Figure 7. Leader Follower experimental geometry, angle measurement and Bird Eye View images showing pixel count at two points for straight and angle maneuvering 
The International Journal of Multimedia \& Its Applications (IJMA) Vol.6, No.5, October 2014

\section{CONCLUSIONS}

The algorithms used for vision detection and localization provide a simple but effective processing approach in real time for the leader-follower formation control approach considered in the paper. The solution is based on pixel variation of the images by referencing to two arbitrarily designated positions in the image frames. The tracking enables a follower to determine its relative position and orientation with respect to the leader in its field of view. Performance of the proposed method has been demonstrated on a laboratory setup composed of two mobile robot platforms. Experimental results demonstrate that overall accuracy of recognition is very good (in some case even $100 \%$ ), thus providing accurate calculation of distance between the leader and the follower. Future work will be toward improvement of robustness of the proposed method with respect to various environment conditions (light intensity, shadows, etc) as well using multiple vehicles for validations.

\section{REFERENCES}

[1] C. Harris and M. Stephens. A combined corner and edge detector. In Alvey vision conference, volume 15,page 50. Manchester, UK, 1988.

[2] J. Shi and C. Tomasi. Good features to track. In IEEE Computer Society Conference on Computer Vision and Pattern Recognition, pages 593-600, 1994.

[3] D.G Lowe. Object recognition from local scale invariant features. In International Conference on Computer Vision, volume 2, pages 1150-1157. Greece,1999.

[4] Malamas. E. N, E G.M Petrakis, Zervakis. M, Petit. L, Jean-Didier Legat (2003). "A survey on industrial vision systems, applications and tools", Image and Vision Computing, 21, 2, 10, 171-188.

[5] Mohamed Aly, (2008) "Real time Detection of Lane Markers in Urban Streets", IEEE Intelligent Vehicles Symposium Eindhoven University of Technology Eindhoven, The Netherlands, 7-12.

[6] Hsu, C.C.; Lu, M.C.; Wang, W.Y.; Lu, Y.Y. (2009) Three dimensional measurement of distant objects based on laser-projected CCD images. IET Sci. Meas. Technol. 3, 197-207.

[7] Lu, M.C.; Wang, W.Y.; Chu, C.Y. (2006) Image-based distance and area measuring systems. IEEE Sens. J, 6, 495-503.

[8] Wang, W.Y.; Lu, M.C.; Kao, H.L.; Chu, C.Y. (2007) Nighttime Vehicle Distance Measuring Systems. IEEE Trans. Circ. Syst. II: 54, 81-85.

[9] Hus, C.C.; Lu, M.C.; Wang, W.Y.; Lu, Y.Y. (2009) Distance measurement based in pixel variation of CCD image. ISA Trans., 40, 389-395. 